\title{
Paraproteinämie bei einem Kind mit variablem Immundefektsyndrom
}

R. Pudill, P. Tarkkanen

Aus dem Laboratorium P. Tarkkanen, Mönchengladbach

\section{Zusammenfassung:}

Ein 11 Jahre alter Junge mit „Allgemeiner, variabler Immundefizienz” (CVID) entwickelte ein IgG/幺, IgG/ $\lambda$-Doppelparaprotein, später trat ein $\mathrm{Ig} G / x$-Paraprotein auf.

Der Nachweis der Monoklonalität erfolgte mittels Immunfixation und Immunelektrophorese.

Die Serumeiweiß-Elektrophorese zeigte einen $M$-Gradienten zwischen dem $\beta$ - und $\gamma$-Globulinbereich. Häufige Ursachen für immunologische Defektsyndrome und Paraproteinämie, wie multiples Myelom, Leukosen, Proteinverlust, Autoimmunerkrankungen, schwere Infektionen, Splenektomie und Thymushypoplasie konnten ausgeschlossen werden, nicht dagegen ein Einfluß der Medikamente Phenytoin und D-Penicillamin.

Die Hyperstimulation einer begrenzten Zahl funktionsfähiger Plasmazell-Klone und kreuzreagierende Klone können die Ursache für das vermehrte Auftreten von Paraprotein bei Immunmangelerkrankungen sein.

Schlüsselwörter:

Paraprotein - monoklonale Gammopathie - Immundefektkrankheiten - Antikörper-Mangelsyndrome humorale Immundefekte - kombinierte Immundefekte - allgemeine variable Hypogammaglobulinämie Immunfixations-Elektrophorese

\section{Summary:}

In an eleven year old boy with common variable immunodeficiency (CVID) a double paraprotein IgG/\%, $\mathrm{IgG/ \lambda}$ was detected. Some time later it changed to a $\mathrm{lg} G / x$-paraprotein. The evidence of monoclonality was done by immunefixation and immune-electrophoresis. The serumprotein-electrophoresis showed a $M$ gradient between the $\beta$ - and $\gamma$-globuline fraction. Common causes for immunological defect syndromes and paraproteinemia like multiple myeloma, some forms of atypical lymphatic leukemia, nephrotic syndrome, autoimmune-diseases, infections, splenectomia and thymus hypoplasia could be excluded, but there remains a possible influence of the drugs Phenytoin and D-Penicillamin.

Hyperstimulation of a limited number of plasmacell clones and cross-reacting clones can be the reason of paraproteinemia in immune defect diseases.

\section{Keywords:}

Paraproteinemia - monoclonal gammopathy - immune deficiency diseases - antibody deficiency syndromes - humoral immune defects - combined immune defects - common variable immunodeficiency immunefixation-electrophoresis

\section{Einleitung}

Paraproteinämie im Kindesalter ist ein äußerst seltener Befund, unabhängig davon, ob sie in Verbindung mit einem multiplen Myelom, einer Leukämie oder einer Immunmangelerkrankung auftritt.

Während das multiple Myelom oder Plasmozytom durch Proliferation meistens eines Plasmazellklons, seltener zweier oder dreier Klone gleichzeitig (44) im Knochenmark, in extramedullärem Gewebe oder Lymphknoten (46) und einen typischen zytomorphologischen Befund des Sternalmarks gekennzeichnet ist, gelten bei einer benignen Paraproteinämie, wie sie im Zusammenhang mit Immunmangelsyndromen auftreten kann, als diagnostische Kriterien nur der M-Gradient in der SerumeiweißElektrophorese und der Nachweis monoklonaler Immunglobuline mittels Immunfixation und/oder Immunelektrophorese, wobei die Immunfixation durch ihre höhere Empfindlichkeit und größere Aussagekraft der Immunelektrophorese überlegen ist $(4,37)$. Zu diesen sekundären Paraproteinämien rechnet man neben den fakultativ paraproteinämischen Hämoblastosen auch die Parapro- teinämien, vergesellschaftet mit Karzinomen, Hepatopathien, schweren Infektionen einschließlich der HIV-Infektion $(i 1,35,45,48,55,58)$ und Autoimmunerkrankungen $(43,56)$. Vereinzelt wurden monoklonale Gammopathien und auch transitorische Paraproteine (47) bei Patienten mit primären Immunmangelerkrankungen, wie z. B. Wiskott-Aldrich-Syndrom (7, 13, 47), schwerem kombinierten Immundefekt $(13,14,18,22,52)$ und Ataxia telangiectasia (10) beschrieben.

Wir berichten über eine Paraproteinämie bei einem 11 Jahre alten Kind mit ,Allgemeiner, variabler Immundefizienz" (common variable immunodeficiency = CVID).

Das variable Immundefizienzsyndrom umfaßt alle Formen des Antikörpermangelsyndroms, die nicht durch eine lymphoretikuläre Systemerkrankung, ein Plasmozytom, einen enteralen oder renalen Proteinverlust, Unterernährung oder eine längerfristige medikamentöse Immunsuppression erklärt werden können, oder genau definierten humoralen oder zellulären Immundefekten zuzuordnen sind. 


\section{Material und Methoden}

\section{Fallbeschreibung}

Der untergewichtige 11 Jahre alte Junge fiel durch rezidivierende sinupulmonale Infektionen auf. Häufig auftretende Ekzeme und mykotische Infektionen der Haut wurden früher als Sonnenallergie oder Fotodermatitis gedeutet.

Wunden an Haut und Schleimhäuten heilen sehr langsam und entzünden sich öfters. Bei bekanntem zerebralen Anfallsleiden (Grand-mal-Epilepsie) steht das Kind seit 5 Jahren unter ständiger Phenytoin- und Valproinat-Medikation.

Seit ein bis zwei Jahren wird der Junge als äußerst aggressiv, ja sogar selbstzerstörerisch bezeichnet.

In der Familie sind sonst keine Fälle von Immundefektsyndromen oder Immunmangelerkrankungen bekannt bzw. aufgetreten.

\section{Immunglobuline}

Die Immunglobulinbestimmung erfolgte nephelometrisch mit einem Immuno-Chemistry-System (ICS)-Rate Nephelometer, Firma Beckman Instr. mit monospezifischen Antisera der Firma Beckman.

\section{Quantitative Leichtketten-Bestimmung}

Die Bestimmung der Leichtketten am ICS-Rate Nephelometer der Firma Beckman Instr. erfolgte mit Antisera der Firma·Kallestad Lab. Inc., Quantimetric ${ }^{\circledR}$.

\section{Serumeiweiß-Elektrophorese}

Die elektrophoretische Trennung erfolgte auf Celluloseazetatfolien der Firma Boskamp in einem Boskamp-Elektrophoresesystem. Angefärbt wurde mit Amidoschwarz 10B. Die Auswertung erfolgte mittels eines Scanners der Firma Hirschmann. Serumeiweiß wurde nach der BiuretMethode bestimmt.

\section{Immunfixations-Elektrophorese}

Die Immunfixation erfolgte auf Agarosefolien mittels des Paragon-Systems der Firma Beckman Instr.

Verwendet wurden folgende Antisera:

Anti-Human-polyvalent

Anti-Human-IgG/ $\gamma$-Kette

(Beckman), (Beckman, Fresenius, Kallestad),
Anti-Human-Ig/L-Kette. Typ Kappa und Typ Lamda (Beckman, Behring, Kallestad),

Anfärbung mit Coomassie-Brillantblau R-250.

\section{Immunelektrophorese}

Die immunelektrophoretischen Analysen wurden auf Folien der Firma Corning mit monospezifischen Antisera der Behringwerke, Marburg, durchgeführt, angefärbt wurde mit Amidoschwarz 10B.

\section{Immunologische Untersuchungen}

Die Bestimmung des Prozentsatzes von Zellen mit T4Oberflächenantigen und Zellen mit T8-Oberflächenantigen im peripheren Blut erfolgte mittels indirekter Immunfluoreszenz unter Verwendung monoklonaler Antikörper OKT4 und OKT8 durch mikroskopische Differenzierung.

OKT4, OKT8: Ortho-mune 4-, Ortho-mune 8-Lymphozytenisolierung, Firma Ortho.

Die Bestimmung des Prozentsatzes von T-Zellen, B-Zellen und Null-Zellen im peripheren Blut erfolgte mikroskopisch nach Lymphozytenisolierung und unter Verwendung von monoklonalen T-Zell-Antikörpern: Quantigen T \& B-Zellen Assay, Bio Rad Lab.

\section{Zirkulierende Immunkomplexe}

Nach Fällung der Immunkomplexe mit Polyethylenglykol, Waschen und Wiederauflösen wurden die Proteine mittels Immundiffusion bestimmt: ClC-Test, Screening IgG/ $\lg M$ und Profil IgG, IgA, IgM, C3c, C1q, Boehringer Ingelheim Diagnostika GmbH.

\section{Differentialblutbild}

Die Erstellung des Blutbildes einschließlich der zytochemischen und zytometrischen Differenzierung der Leukozyten erfolgte mit einem Hämatologie-Automaten, dem Technicon- $\mathrm{H} 1$-Analysator, Firma Technicon $\mathrm{GmbH}$, Bad Vilbel.

\section{HIV-Antikörpertest}

HTLV-III/LAV-Antikörper, Vironostika, Mikroelisa-System, Firma Organon Teknika.

Tab. 1: Immunglobulin-Konzentrationen, Serumeiweiß-Elektrophoresen, Komplemente 3 und 4 bei einem Kind mit Paraproteinämie und Immundefizienz über einen Zeitraum von 2,5 Monaten

\begin{tabular}{|c|c|c|c|c|c|c|}
\hline Parameter (Einheit) & Normalbereich & 8. 12.1986 & 16. 12.1986 & 9.1.1987 & 10. 2. 1987 & 25. 2. 1987 \\
\hline $\begin{array}{l}\lg A(\mathrm{mg} / \mathrm{dl}) \\
\lg M(\mathrm{mg} / \mathrm{dl}) \\
\operatorname{lgg}(\mathrm{mg} / \mathrm{dl})\end{array}$ & $\begin{array}{r}50-220 \\
50-220 \\
700-1800\end{array}$ & $\begin{array}{c}12,4 \\
30,6 \\
580\end{array}$ & $\begin{array}{l}12,8 \\
25,9 \\
534\end{array}$ & $\begin{array}{l}13,3 \\
31,4 \\
567\end{array}$ & $\begin{array}{l}15,5 \\
34,7 \\
569\end{array}$ & $\begin{array}{l}15,0 \\
22,8 \\
634\end{array}$ \\
\hline $\begin{array}{l}\text { C3 (mg/dl) } \\
\text { C4 (mg/dl) }\end{array}$ & $\begin{array}{r}80-120 \\
10-40\end{array}$ & $\begin{array}{l}158 \\
46,4\end{array}$ & $\begin{array}{r}111 \\
20\end{array}$ & $\begin{array}{r}122 \\
28\end{array}$ & $\begin{array}{l}128 \\
26,7\end{array}$ & $\begin{array}{r}130 \\
28\end{array}$ \\
\hline $\begin{array}{l}\text { Ges.Eiw. (g/l) } \\
\text { Alb. (rel. \%) } \\
\alpha_{1} \text {-Glob. (rel.\%) } \\
\alpha_{2} \text {-Glob. (rel.\%) } \\
\beta \text {-Glob. (rel.\%) } \\
\gamma \text {-Glob. (rel.\%) }\end{array}$ & $\begin{aligned} 65- & 85 \\
54- & 68 \\
2,5- & 4,5 \\
6,5- & 10,0 \\
8,0- & 15,0 \\
11,0- & 19,0\end{aligned}$ & $\begin{array}{r}56 \\
80,8 \\
1,9 \\
5,3 \\
7,8 \\
4,2\end{array}$ & $\begin{array}{r}66 \\
69,9 \\
4,5 \\
7,5 \\
11,5 \\
6,6\end{array}$ & $\begin{array}{r}68 \\
76,8 \\
2,7 \\
6,9 \\
9,0 \\
4,6\end{array}$ & $\begin{array}{r}67 \\
69,9 \\
4,0 \\
9,7 \\
11,7 \\
4,7\end{array}$ & $\begin{array}{r}65 \\
72,1 \\
2,9 \\
7,8 \\
10,5 \\
6,7\end{array}$ \\
\hline
\end{tabular}




\section{Ergebnisse}

\section{Serumeiweiß-Elektrophorese}

Die Serumeiweiß-Elektrophoresen zeigen einen relativ breitbasigen Gradienten im $\beta-\gamma$-Globulin-Zwischenbereich bei einer gleichzeitig sehr niedrigen $\gamma$-Globulinfraktion. Die quantitative Bestimmung des monoklonalen Immunglobulins ergab $200 \mathrm{mg} / \mathrm{dl}$ (3-4 rel.\%), also etwa die Hälfte der gesamten $\gamma$-Globulinfraktion (Abb. 1, 2 und Tab. 1). Die Paraproteinkonzentration nimmt im Laufe des Untersuchungszeitraumes ab. Ende Februar beträgt sie nur noch $120 \mathrm{mg} / \mathrm{dl}$, Mitte März ist ein M-Gradient nicht mehr darstellbar (Abb. 6).

Der M-Gradient gab den Anlaß für weitergehende immunologische Untersuchungen, wie Immunelektrophorese und Immunfixation.

\section{Immunglobuline}

Die Immunglobulinkonzentrationen bleiben für $\lg A$ und IgM über den gesamten Untersuchungszeitraum stark, für IgG mäßig erniedrigt. Eine Tendenz ist nicht erkennbar (Tab. 1).

Komplement 3 liegt unbedeutend oberhalb, Komplement 4 innerhalb des entsprechenden Normbereiches.

Die Konzentrationen der zirkulierenden Immunkomplexe, IgG und IgM, liegen innerhalb der entsprechenden Normbereiche.

\section{Leichtketten-ratio}

Das mehrfach bestimmte Leichtkettenverhältnis $L / \chi: L / \lambda$. beträgt im Mittel 1,80 [1,35-2,69].

\section{Immunelektrophorese}

Das IgG - Präzipitat zeigt im Vergleich zu einem Normalserum auffällige Veränderungen, $L / x$ und $L / \lambda$ stellen sich dagegen normal dar (Abb. 5).

\section{Immunfixation}

Die Immunfixations-Elektrophorese zeigt im Januar 1987 diskrete Banden für IgG, $L / \varkappa$ und $L / \lambda$. Die Ergebnisse

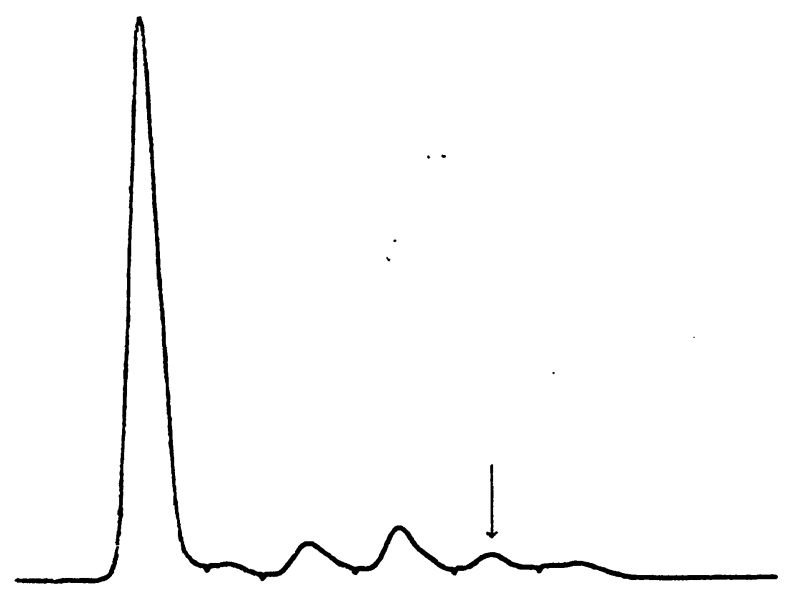

Abb. 1 : Serumeiweiß-Elektrophorese, Januar 1987. Der M-Gradient ist mit einem Pfeil markiert waren mit Antisera verschiedener Hersteller über 3 Wochen reproduzierbar (Abb. 3 ). Im Februar waren in der Immunfixation diskrete Banden nur noch im Falle von IgG und L/x (Abb. 4), Mitte März nicht mehr darstellbar.

\section{Lymphozytenpopulation und T4/T8-Verhältnis}

Es zeigt sich eine normale Verteilung der $\mathrm{B}-, \mathrm{T}$ - und $\mathrm{O}$ Lymphozyten: B-Zellen 18\% [5-15]; T-Zellen 70\% [6085]; 0 -Zellen $12 \%$ [8-14].

Das Verhältnis T-Helfer- zu T-Suppressorzellen ist jedoch mit $0,9[1,7 \pm 0,2]$ deutlich vermindert.

\section{AK-HIV (Elisa)}

Der HIV-Antikörpertest wurde während des Beobachtungszeitraumes mehrfach mit negativem Ergebnis durchgeführt.

\section{Hämatologische Befunde}

Bei der mehrfach automatisch erstellten Blutbildanalyse ergab sich folgendes Bild:

Leukozyten $\left(\cdot 10^{3} / \mu \mathrm{l}\right)=5,9 / 7,4 / 6,1[8-12]$;

Erythrozyten $\left(\cdot 10^{6} / \mu \mathrm{l}\right)=4,7 / 5,5 / 4,8[4,5-5,5]$;

Hämoglobin $(\mathrm{g} / \mathrm{dl})=13,5 / 15,5 / 14,4$ [13-16];

Hämatokrit $(\%)=38,8 / 46,8 / 39,3[36-46]$;

Thrombozyten $\left(\cdot 10^{3} / \mu \mathrm{l}\right)=202 / 257 / 282[150-400]$;

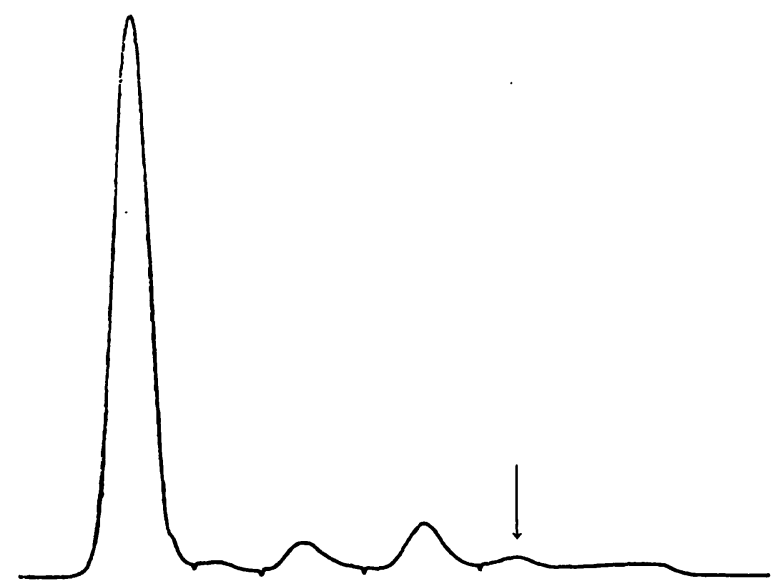

Abb. 2: Serumeiweiß-Elektrophorese, Ende Februar 1987. Der $M$-Gradient ist mit einem Pfeil markiert

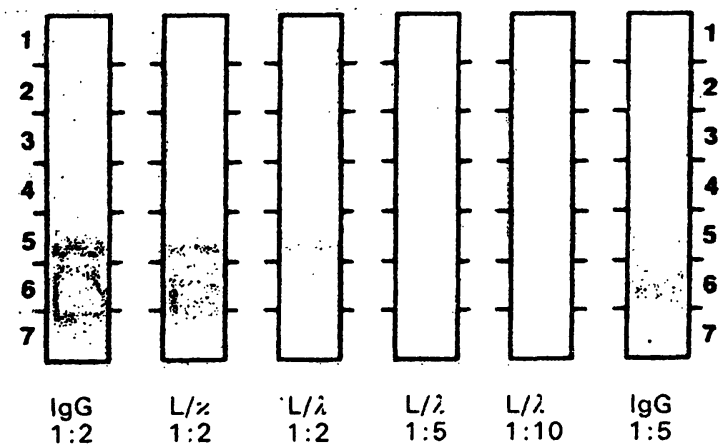

Abb. 3: Immunfixations-Elektrophorese, Januar 1987. IgGx/ IgGi-Doppelparaprotein 
Lymphozyten $(\%)=64 / 49 / 37,6[25-40]$;

Lymphozyten $\left(\cdot 10^{3} / \mu 1\right)=3,67 / 3,18[1,5-4,1]$;

Monozyten $(\%)=8 / 9 / 11,5[2-6]$;

Eosinophile Gran. $(\%)=8 / 5 / 3,3[2-4]$;

Basophile Gran. (\%) $=1,3 / 1,2[0-1]$.

Auffallend ist der hohe Anteil der Monozyten und Lym. phozyten. Die Blutsenkungsgeschwindigkeit bleibt während des gesamten Beobachtungszeitraumes innerhalb des Normbereiches: 2/5,3/7,2/5 mm.

Folgende Parameter aus Serum bzw. Plasma wurden zusätzlich bestimmt:

$\lg E(R \mid A)=27,3 \mathrm{IU} / \mathrm{mI}$ [bis 120];

Parathormon (RIA) $=0.35 \mathrm{ng} / \mathrm{ml}[0,1-1,3]$;

freies Thyroxin, $\mathrm{FT}_{4}(\mathrm{RIA})=1,3 \mu \mathrm{g} / \mathrm{ml}[0,1-1,3]$;

freies Trijodthyronin, $\mathrm{FT}_{3}(\mathrm{RIA})=6,2 \mathrm{ng}[2,7-6,8]$;

Thyreotropes Hormon, supersensitiv, TSH basal (RIA) = $1.7 \mu \mathrm{U} / \mathrm{ml}[0,4-4,01]$;

Carcino embryonales Antigen, CEA (RIA) $=<1,0 \mathrm{ng} / \mathrm{ml}$ $[<5]$;

Tissue Polypeptide Antigen, TPA (RIA) $=59,5 \mathrm{U} / \mathrm{I}$ $[<120]$;

C-reaktives Protein, $\mathrm{CRP}=<0,6 \mathrm{mg} / \mathrm{dl}[<0,8]$;

Rheumafaktor quant., RF $=<60 \mathrm{IU} / \mathrm{ml}$ [bis 60$]$;

Waaler-Rose: $1:<20$ [bis $1: 20$ ];

Anti-Streptolysin $-0=100 \mathrm{IE} / \mathrm{ml}$ [bis 200];

Auto-AK Zellantigen, Antinukleäre $\mathrm{AK}, \mathrm{ANF}=$ negativ;

Auto-AK Anti-ds DNA (RIA) $=1,0 \mathrm{mg} / \mathrm{l}$ [bis 5];

AK Cytomegalie, CMV (ELISA) IgG = negativ, $\lg M=$ negativ;

AK Epstein-Barr Virus, EBV (ELISA), IgG = negativ, $\lg M=$ negativ;

GOT $=8 \mathrm{U} / \mathrm{l}$ [bis 18]; GPT $=5 \mathrm{U} / \mathrm{l}$ [bis 22];

$\gamma-\mathrm{GT}=10 \mathrm{U} / \mathrm{l}$ [bis 34];

Harnstoff $=30 \mathrm{mg} / \mathrm{dl}[$ bis 50$] ;$ Harnsäure $=5,3 \mathrm{mg} / \mathrm{dl}$ [bis 7,0];

Creatinin $=0,5 \mathrm{mg} / \mathrm{dl}$ [bis 1,2$]$;

Blutzucker nüchtern: $76 \mathrm{mg} / \mathrm{dl}$ [50-100];

Eisen $=129 \mu \mathrm{g} / \mathrm{dl}[80-150]$;

Kalzium ionisiert $=0,79 \mathrm{mmol} / \mathrm{l}[0,83-1,25]$;

Kalzium gesamt $=2,4 \mathrm{mmol} / \mathrm{l}[2,25-2,7]$;

anorg. Phosphor $=1,9$ und $1,5 \mathrm{mmol} / \mathrm{l}[0,8-1,6]$;

Natrium $=141 \mathrm{mmol} / \mathrm{l}[135-159]$;

Zink $=0,91 \mu \mathrm{g} / \mathrm{ml}[0,7-1,3]$;

Thromboplastinzeit, part. $=37,6$ und $37,2 \mathrm{sec}$. [bis 40].

Mehrfach im Untersuchungszeitraum wurde der Serumspiegel des Antikonvulsivums Natrium-Valproinat (2Propyl-Valeriansäure, Natriumsalz; Dipropylessigsäure, Natriumsalz) bestimmt, der Wert bewegt sich zwischen 55 und $76 \mu \mathrm{g} / \mathrm{ml}$ [therapeutischer Bereich: 50-100].

Urinbefund:

Urin-Eiweiß = 26,6 mg/l [bis $150 \mathrm{mg} / \mathrm{die}$ ];

Bence-Jones-Kochprobe: negativ;

Immunfixations-Elektrophorese $=$ Bence-Jones- $x$-Kette $=$ negativ,

Bence-Jones- $\lambda$-Kette $=$ negativ .

\section{Diskussion}

Defektimmunopathien werden unterteilt in angeborene (primäre) und erworbene (sekundäre) Immundefektkrankheiten $(19,50,54)$. Primäre Immunmangelzustände beruhen zu $90 \%$ auf einer Störung des humoralen und/ oder zellulären Immunsystems; etwa $10 \%$ betreffen die Phagozytose und weniger als $1 \%$ das Komplementsystem. Bei den Störungen der humoralen Immunität .(B-Zell-

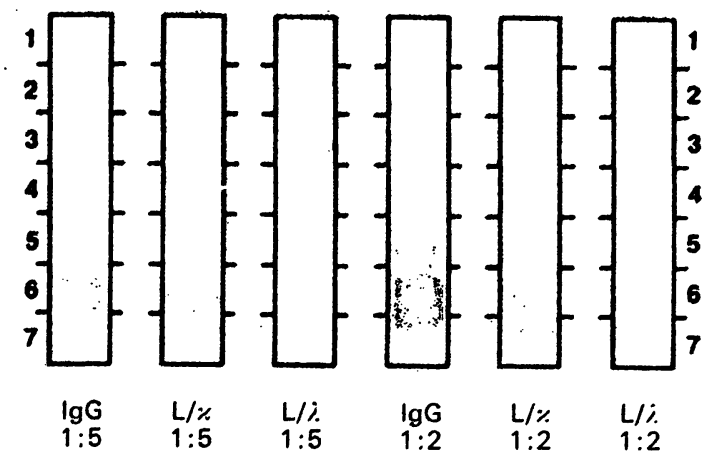

Abb. 4: Immunfixations-Elektrophorese, Ende Februar 1987 IgG/\%-Paraprotein

$N$

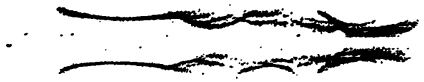

$\mathrm{AH}$

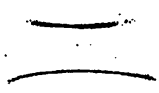

N

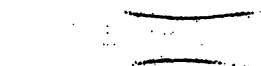

P

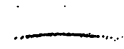

L/

$\mathrm{N}$

Abb. 5: Immunelektrophorese. $N=$ Normalserum, $P=$ Patient $A H=$ Anti-Human-polyvalent, IgG $=$ Anti-Human-lgG/ $\%$ Kette, $L / \varkappa=$ Anti-Human-Ig/L-Kette Typ Kappa, $L / \lambda=$ AntiHuman-Ig/L-Kette Typ Lambda

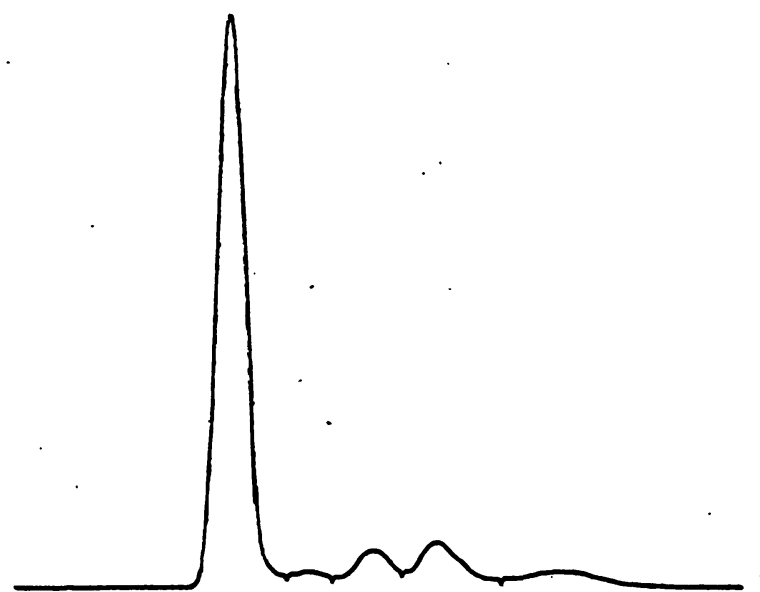

Abb. 6: Serumeiweiß-Elektrophorese, Mitte März 1987

Defekte) unterscheidet man die Agammaglobulinämie (Morbus Bruton) - periphere Zellen fehlen hierbei völlig -, den selektiven IgA-Mangel, mit einer Inzidenz von 1:500 bis $1: 700$ in der Normalbevölkerung der häufigste Immundefekt (30), die transitorische Hypogammaglobu- 
linämie mit einer wesentlich verzögerten eigenen Immunglobulinproduktion beim Säugling, den seltenen selektiven IgM-Defekt und das Hyper-IgM-Syndrom.

Die Serumimmunglobulinbestimmung ist die klinisch relevanteste Screeningmethode zur Erfassung eines humoralen Immundefektes. Erste Hinweise auf das Vorliegen einer Hypogammaglobulinämie gibt auch schon die Serumeiweiß-Elëktrophorese.

Kinder mit humoralem Immundefekt leiden oft unter chronisch rezidivierenden Infektionen der Haut, des Gastrointestinal- und Respirationstraktes. Bakterielle Infektionen sind typisch für B-Zell-, Phagozyten- und Komplementdefekte. Virale Infektionen verlaufen wegen der intakten zellulären Immunität komplikationslos. Zelluläre Immundefekte (T-Zell-Defekte) mit isoliertem Ausfall der TZell-Funktion, wie z.B. beim DiGeorge-Syndrom (kongenitale Thymusaplasie oder-Hypoplasie) sind selten, da der T-Zell-Defekt aufgrund der für die Antikörperproduktion notwendigen $T$ - und $B$-Zell-Interaktion mit einer abnormalen $B$-Zell-Immunität verbunden ist $(15,49)$.

Die Serumwerte für die Immunglobuline $\lg A, \lg M$ und IgG liegen in der Regel im Normbereich. Die Antikörperproduktion gegen alle Antigene, bei denen eine T-B-ZellKooperation erforderlich ist, sind jedoch gestört.

Im Blut sind bei nicht verminderter Gesamtlymphozytenzahl zu $80 \%$ B-Lymphozyten nachweisbar. T-Lymphozyten sind meist vorhanden, jedoch ist ihre Funktionsfähigkeit erheblich eingeschränkt (16). Beim DiGeorge-Syndrom ist auch die Entwicklung der Nebenschilddrüsen gestört, so daß die Diagnose durch folgende Befunde erhärtet werden kann. Parathormon ist oft nicht nachweisbar oder sehr niedrig, Kalzium ist erniedrigt, Phosphat erhöht. Für T-Zell-Defekte sind Infektionen mit Viren, Pilzen (besonders Candida) und gering pathogenen Bakterien und Protozoen (z. B. Pneumocystis carinii) typisch. Die allergischen Spätreaktionen durch Hauttestung mit bestimmten Antigenen fallen ebenso wie der Stimulierungsversuch mit Dinitrochlorbenzol negativ aus.

Bei den immunologischen Defektsyndromen mit kombiniertem humoralem und zellulärem Immuñdefekt können unterschiedliche Störungen der B- und T-Zell-Entwicklung zu den entsprechenden klinischen Symptomen führen.

Beim schweren kombinierten Immundefekt (SCID) liegt primär eine retikuläre Dysgenesie (Fehlen der lymphomonozytoiden Stammzellen) zugrunde $(6,8)$. Im peripheren Blut ist die extreme Lymphopenie, sowie das Fehlen der Immunglobuline am auffälligsten. Klinisch stehen gastrointestinale Infekte im Vordergrund. Weitere kombinierte Immundefekte sind das Louis-Bar-Syndrom, verbunden mit Ataxia telangiectasia und das Wiskott-AldrichSyndrom (Immunmangel verbunden mit Thrombozytopenie und Ekzem) (51).

Teils den humoralen, teils den kombinierten Immundefekten zugerechnet wird die heterogene Gruppe der sogenannten „Allgemeinen, variablen Hypogammaglobulinämie (CVID)" $(18,38)$. Diese Erkrankungsform wird erst im späten Kindesalter manifest. Ursächlich scheint eine Reifestörung des B-Lymphozyten zur Immunoglobulinesezernierenden Plasmazelle vorzuliegen (18). Bei etwa $30 \%$ können T-Zell-Defekte nachgewiesen werden, zum Teil liegt eine Erhöhung der T-Suppressor-Zellen vor. Andere Patienten zeigen einen verminderten Anteil T-Helfer-Zellen oder eine beeinträchtigte T-Helferzell-Funktion $(6,18,23)$. Die Dysimmunoglobulinämie betrifft ver- schiedene Immunglobuline. Die B-Zell-Zahl im Blut ist jedoch nicht vermindert. Die Immunantworten vom verzögerten Typ verlaufen im allgemeinen normal. Neben einer Häufung von viralen und bakteriellen Infektionen entwickeln sich häufig Lymphome und Leukämie. Auffällig ist auch die hohe Inzidenz von begleitenden Autoimmunerkrankungen.

Sekundäre Immundefizienzen $(19,50,54)$ können verursacht sein durch neoplastische Prozesse des lymphoretikulären Systems, wie Morbus Hodgkin, multiples Myelom und Leukosen. In einem hohen Prozentsatz der Patienten mit multiplem Myelom werden sekundäre Immunglobulin-Mangelsyndrome beobachtet; in etwa der Hälfte aller Fälle von chronisch lymphatischer Leukämie kommt es zu einer erheblichen Verminderung der Immunglobulinsynthese.

Weitere Ursachen für sekundären Immunmangel sind:

Proteinverlust bzw. mangelnde Proteinzufuhr, wie exsudative Gastroenteropathie, Mukoviszidose, nephrotisches Syndrom (17), Bence-Jones-Proteinurie, Verbrennungen;

Splenektomie, Sichelzellanämie, Diabetes mellitus, Urämie, Sarkoidose;

Autoimmunerkrankungen (rheumatische Arthritis, Lupus erythematodes, multiple Sklerose usw.).

Autoimmunerkrankungen können durch eine Fehlregulation der humoralen oder zellulären Immunantwort entstehen.

Auslöseereignisse können sein:

Kreuzreaktionen von Antikörpern;

Infektionen durch Epstein-Barr-, Cytomegalie- und Herpesviren, sowie HIV;

Medikamente, z. B. Zytostatika, Steroide, Phenytoin und D-Penicillamin (50);

Drogen und sog. Umweltgifte, wie Chlorkohlenwasserstoffe, Pestizide, Schwermetalle; ionisierende Strahlen und die Applikation radiomimetischer Präparate.

Das relativ häufige Auftreten von Paraprotein bei Immunmangelerkrankungen ist möglicherweise auf eine Hyperstimulation der nur begrenzt zur Verfügung stehenden bzw. funktionsfähigen Plasmazellklone oder T-Lymphozyten zurückzuführen.

Fakultative Paraproteinämien werden beschrieben bei chronisch lymphatischer Leukämie $(11,12)$, chronischer Lymphadenose (53), Retikulumzellsarkom (32), malignem Lymphom, Amyloidose (39), Hepatopathien (48), Karzinomen $(29,31)$, schweren Infektionen (55), Malabsorptions-Syndrom $(20,21)$, nephrotischem Syndrom (41) und anderen Grundleidèn sowie bei Autoimmunerkrankungen.

Immundefekte begünstigen die Onkogenese ebenso wie schwere Infekte.

Sekundärfaktoren sind zudem neben mutationsähnlichen Veränderungen für die Art des Tumors, seine Malignität sowie seine Metastasierung in anderen Geweben von Bedeutung. So spielt das Epstein-Barr-Virus eine Rolle bei der Bildung von B-Zell-Lymphomen oder Plasmozytomen (31). Charakteristischerweise werden oft das Burkitt-Lymphom - sonst eine Erkrankung des Kindesalters - und Non-Hodgkin-Lymphome bei AIDS-Patienten beobachtet $(59,60)$.

Das Auftreten von Paraproteinämien bei Personen mit HIV-Infektion wurde von uns kürzlich beschrieben (45). 
Die Autoimmunerkrankung multiple Sklerose geht mit der Bildung von autochthonen, oligoklonalen Liquor-Immunglobulinen einher $(42,43)$. S. $H$. Tsung berichtet über monoklonale Gammopathie im Zusammenhang mit MS (56).

Schwere Erkrankungen können - insbesondere in Verbindung mil einer sekundären Immunmangelerkrankung - auch bei Kindern zu Paraproteinämie führen (27). $\mathrm{M}$. Tichý et al. (55) beschrieben ein transitorisches lgM/ $\lambda$-Paraprotein bei einem 15 Monate alten Kind nach einer schweren Mittelohr-Entzündung, F. Danon und M. Seligmann (13) nach immunsuppressiver Therapie, bei Hämoblastosen, sowie infolge sekundärer Immundefektsyndrome, A. J. Piek et al. bei Amyloidose (39), T. Groshong et al. nach einer chronischen CMV-Infektion (22) und $Z$. A. Zawadzki et al. nach Virus-Pneumonie (58).

Als diagnostische Kriterien einer benignen Folge-Paraproteinämie gelten der Nachweis von monoklonalem Immunglobulin und der $\mathrm{M}$-Gradient in der SerumeiweißElektrophorese. Zytomorphologische Veränderungen sind nicht nachweisbar. Das Verhältnis $L / \%: L / \lambda$ ist nicht verschoben.

Paraproteinämie oder auch Bence-Jones-Proteinurie bei primären Immunmangelerkrankungen wird beschrieben bei $\operatorname{SCID}(9,13,14,18,22,52)$, Wiskott-Aldrich-Syndrom $(7,13,47)$ und bei Ataxia telangiectasia (10).

Paraproteinämie und/oder Bence-Jones-Proteinurie ist obligat beim multiplen Myelom oder Plasmozytom.

Multiple Myelome, insbesondere extramedulläre, solitäre und Lymphknoten-Plasmozytome im Kindesalter sind extrem selten $(36,40,46)$. K. Kruse, H. Stein, W. H. Hitzig et al. (34) berichten ausführlich über klinische und immunologische Daten bei einem 10 Jahre alten Mädchen mit Lymphknoten-Plasmozytom und $\lg \mathrm{A} / x$-Paraproteinämie. S. C. Bernstein et al. (5) fanden bei einem 12 Jahre

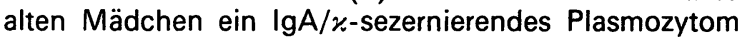
im Nasopharynx, G. E. Hartmann und C. D. Baroni beschreiben solitäre, sekretorische Plasmozytome der Lunge im Kindesalter $(3,24)$ und.G. M. Hewell multiple Myelome bei Patienten im Alter von 17 bis 22 Jahren (26).

Als diagnostische Kriterien einer malignen Paraproteinämie gelten außer dem Nachweis von monoklonalem, bioder oligoklonalem Immunglobulin mittels Immunelektrophorese und/oder Immunfixations-Elektrophorese und dem M-Gradient in der Serumeiweiß-Elektrophorese auch der zytomorphologische Befund des Sternalmarks. Der röntgenologische Nachweis von Osteolysen ist als richtungsweisender Ergänzungsbefund zu werten. In den allermeisten Fällen geht mit Ausnahme des L-KettenMyeloms, bei dem meist die Immunglobulinklassen A, M und $G$ erniedrigt sind $(28,57)$, das multiple Myelom mit einer Erhöhung einer, manchmal auch zweier Immunglobulinklassen und einer signifikanten Verminderung der korrespondierenden Klassen einher.

\section{Ganz allgemein kann man sagen:}

Primäre oder sekundäre Immundefizienz erhöht die Prävalenz zur Paraproteinämie, während obligate Paraproteinämie eine Immundefizienz der nicht betroffenen Immunglobulinklassen zur Folge hat.

In unserem Falle geben die immunologischen, radioimmunologischen, serologischen und hämatologischen Untersuchungen sowie die Anamnese folgendes Bild:
Die Häufung von viralen und bakteriellen Infektionen, vermehrte Affektionen der Haut und Schleimhäute, die deutliche Verminderung der absoluten Zahl der T-HelferZellen bei erniedrigtem Verhältnis von T-Helfer zu T-Suppressor-Zellen und normaler Gesamtlymphozytenzahl, die Bestimmung der T-, B- und Null-Zellen im peripheren Blut, die mäßig erniedrigte IgG - und die stark verminderten IgA- und IgM-Konzentrationen, die stark erniedrigte $\gamma$-Globulinfraktion in der Serumeiweiß-Elektrophorese und die normale Auslösung der Überempfindlichkeitsreaktionen vom Spättyp mit bestimmten Antigenen ermöglichen eine Zuordnung des Immundefektes in die Gruppe der „Allgemeinen, variablen Hypogammaglobulinämien, CVID".

Die Bestimmung der absoluten und relativen Anzahl der T- und B-Lymphozyten ist eine Hilfe für die Erkennung einer Vielzahl von immunologisch bedingten Erkrankungen, beispielsweise bei der Identifikation des Zelltyps von Lymphomen und Leukämien sowie bei Immunmangelerkrankungen. $80 \%$ der Fälle von Non-Hodgkin-Lymphomen leiten sich von B-Zellen ab. Die x-linked Agammaglobulinämie und andere Dysgammaglobulinämien finden sich oft in Verbindung mit einer verminderten Zahl von B-Lymphozyten, die zellverminderte Immunität ist jedoch in der Regel intakt. Das SCID zeichnet sich dagegen durch einen Defekt der Stammzellebene aus; T-Zellen fehlen oft völlig oder deren Zahl ist stark vermindert, der Anteil der B-Zellen ist meistens erhöht.

Der Nachweis zirkulierender Immunkomplexe stellt ein zusätzliches diagnostisches Hilfsmittel für einige sekundäre Immundefizienzen auslösende Erkrankungen dar.

In unserem Fall konnten Defekte der T-Zellreihe, Erkrankungen mit gestörter Phagozytosefunktion und Komplementdefekte sowie kombinierte Defekte, wie SCID, das Louis-Bar- und Wiskott-Aldrich-Syndrom ausgeschlossen werden.

Ein extramedulläres Plasmozytom lag nicht sowie eine schwere Erkrankung, die sekundär eine Paraproteinämie bedingen könnte, lag ebenfalls nicht vor.

Der Nachweis von Paraprotein erfolgte aufgrund eines $M$-Gradienten in der Serumeiweiß-Elektrophorese (Abb. 1, 2) mittels Immunelektrophorese (Abb. 5) und Immunfixation Anfang Januar 1987. Drei Wochen lang war ein IgG-Paraprotein, Typ $\varkappa$ und Typ $\lambda$, also ein Doppelparaprotein, darstellbar (Abb. 3), in der Folgezeit nur noch ein $\operatorname{lgG} / \varkappa$-Paraprotein (Abb. 4).

Mitte März 1987 waren ein M-Gradient und diskrete Banden in der Immunfixation nicht mehr darstellbar (Abb. 6).

Die $\% / \lambda$-Ratio lag während der gesamten Beobachtungszeit innerhalb des Normbereiches, was als Hinweis auf eine benigne Paraproteinämie gewertet werden kann.

Transitorische Paraproteine wurden in der Literatur bisher nur infolge schwerer Infektionserkrankungen (25, 47 , $55)$, beim Wiskott-Aldrich-Syndrom (47) nach zytostatischer Behandlung, bei malignen Lymphomen und Leukämie beschrieben. Bei der Diskussion dieses Falles darf nicht außer acht gelassen werden, daß der Junge seit Jahren an zerebralen Krampfanfällen leidet und mit Phenytoin, Carbamazepin und Valproinat behandelt wird. Aufgrund seiner Infektionsanfälligkeit erfolgte öfters Medikation mit D-Penicillamin. Eine Behandlung mit Phenytoin und/oder D-Penicillamin kann Immunmangelzustände auslösen oder verstärken (50). 
Unsere eigenen Arbeiten und Literaturstudien lassen den Schluß zu, daß Paraproteinämie bei primären und sekundären Immunmangelerkrankungen im Erwachsenenalter relativ häufig zu beobachten ist: etwa 1\% aller Personen über 60 Jahre, $3 \%$ der über 70jährigen und $18 \%$ der über 90 jährigen weisen Paraproteinämie auf. Eine niedrige $\mathrm{Pa}$ raprotein-Konzentration und die normale $x / \lambda$-Ratio im Zusammenhang mit der Abwesenheit von Bence-JonesProtein und zytologischen Abnormalitäten kennzeichnen ihren meist benignen Charakter. Es kann aber - manchmal erst nach Jahren - zur malignen Entartung eines zur Synthese von Immunglobulinen befähigten B-Zellklons kommen (1). Paraproteinämie im Kindesalter ist jedoch ein seltener Befund.

Die Bildung von monoklonalem Immunglobulin läßt sich so erklären, daß infolge eines Defektes des Immunsystems eine spezifische Antikörperantwort wegen einer selektiven Defizienz einer Reihe von Zellklonen nicht stattfinden kann und es zu einer Aktivierung anderer kompensatorischer Mechanismen, z.B. kreuzreagierender Klone, kommt," auch wenn dadurch nur verstärkt unwirksame Antikörper zur Eliminierung potentiell pathogener Antigene gebildet werden.

Für die wissenschaftlichen Diskussionsbeiträge gilt unser besonderer Dank folgenden Kollegen: Prof. Dr. W. H. Hitzig, Universitäts-Kinderklinik Zürich; Prof. Dr. H. Schulte-Wissermann, Kinderklinik, Städt. Krankenanstalten Krefeld; Dr. med. C. U Scherer, Neuss 22; Oberarzt Dr. med. N. Veiser, Kinderstation, Krankenanstalten Neuss, Lukaskrankenhaus. Für die Durchführung der immunologischen Untersuchungen danken wir Herrn Thomas Berche.

\section{Schrifttum:}

1. AXELSSON, M. HÄLLEN, J.: Review of 54 subjects with monoclonal gammopathy. Brit. J. Haemat. 15. 417 (1968).

2. AZAR, H. A., HILL, W. T., OSSERMANN, E. T.: Malignant lymphoma and lymphatic leukemia associated with myeloma-type serum proteins. Amer. J. Med. 23, 239 (1957).

3. BARONI, C. D. MINEO, T. C., RICCI, C. et al.: Solitary secretory plasmacytoma of the lung in a 14 year old boy. Cancer 40, 2329 (1977).

4. BAUS, M., MÜLLER, T., THOMAS, L.: Immunfixations-Elektrophorese zum Nachweis monoklonaler Gammopathien: Durchführung, Interpretation. Fehlermöglichkeiten. Lab.med. 10, 192-200 (1986).

5. BERNSTEIN, S. C., PEREZ-ATAYDE, A. R., WEINSTEIN, H. J.: Multiple myeloma in a child. Cancer 56, 2143-2147 (1985).

6. von BISMARCK, U., PEEST, D. DRÄGER, R., SERBIN, A. et al.: Terminale B

Zellreitung und Immunglobulinsynthese in vitro bei primären und sekundären Immundefekten. Immun. Infekt. 12, 75-87 (1984).

7. BRUCE, R. M., BLAESE, M.: Monoclonal gammopathy in the Wiskott-Aldrich Syndrome. J. Pediatr. 85, 204 (1974).

8. BUCKLEY, R. H. GILBERTSEN, R. B., SCHIFF, R. J. et al.: Heterogeneity of lymphocyte subpopulations in severe combined immunodeficiency. J. Clin. Invest. 58 130 (1976)

9. BUSHELL. A. C., WHICHER, J. T., YUILLE, T.: The progressive appearance of multiple urinary Berice-Jones proteins and serum paraproteins in a child with immune deficiency. Clin. Exp. Immunol. 38, 64-69 (1979).

10. CAWLEY, J. SCHENKEN, J.: Monoclonal hypergammaglobulinaemia of the $\lg M$ type in a 9 year old girl with ataxia telangiectásia. Amer. J. Clin. Path. 54, 790 (1970). 11. CEJKA, J., BOLLINGER, R. O., SCHUIT, H. et al.: Macroglobulinaemia in a child with acute leukemia. Blood 43/2, 191-199 (1974).

12. CONE. L. UHR, J. W.: Immunological deficiency disorders associated with chronic lymphatic leukemia and multiple myeloma. J. Clin. Invest. 43, 2241 (1964).

13. DANON $F$ SELIGMANN $M$. Serum monoclonal immunoglobulins in childhood. Arch. Dis. Child. 48, 207 (1973).

14. De FAZIO, S. R., CRISWELL, B. S., KIMZEY, S. L. et al.: A paraprotein in sever combined immunodeficiency disease detected by immunoelectrophoretic analysis of plasma. Clin. Exp. Immunol. 19, 563-570 (1975).

15. DIGEORGE, A. M. Congenital absence of the thymus and its immunological consequences: Concurrence with congenital hypoparathyroidism. in: Bergsma, $D$. Good. R. A. (eds.) Immunological deficiency in man. Birth defects 116 (1968).

16. GAJL-PECZALSKA. K. J., BIGGAR, W. D., PARK, B. H., GOOD, R. A.: The lymphocytes in DiGeorge syndrome. Lancet, 1344 (1972)

17. GAUTIER, E., HITZIG, W. H., GUGLER, E., BERTSCHMANN, M.: Zur Frage de humoralen Abwehr beim nephrotischen Syndrom. Helv. Paediat. Acta 3, 277 (1961) 18. GEHA. R. S., SCHNEEBERGER, E., MERLER, E., ROSEN, F. S.: Heterogeneity of "acquired" or common variable agammaglobulinaemia. N. Engl. J. Med. 291.1 (1974) 19. GEIGLER. H.: Immunologische Defektsyndrome. In: Immunologie, GrundlagenKlinik-Praxis. Vorlaender, K. O. (ed.). Georg Thieme Verlag. Stuttgart, New York, 260 (1983).

20. GILBERT, E. F., HARLEY, J. B., AVIDO, V. et al.: Thymoma, plasma cell myelom. red cell aplasia and malabsorption syndrome. Amer. J. Med. 44, 820 (1968).
21. GOOD, R. A., BACH, F. H.: Bone marrow and thymus transplants: cellular engineering to correct primary immunodeficiency. In: Clinical Immunobiology, Bd. 2. Bach F. H., Good, R. A. (eds.). Academic Press, New York (1974).

22. GROSHONG, T., HOROWITZ, S., LOVCHIK, J. et al.: Chronic cytomegalovirus infection, immunodeficiency and monoclonal gammopathy - antigen-driven ma lignancy? Pediatrics 88, 217-223 (1976).

23. GUPTA, S.: Lymphocyte subpopulation measurements. Immunol. Letts. 4, 129 (1982).

24. HARTMANN, G. E., SHOCHAT, S. J.: Primary pulmonary neoplasms of childhood: A review. The Annals of Thoracic Surgery 36, 108-119 (1983).

25. HERMES, C., DAUS, H.. KOHLER, M. et al.: Transitorische oligoclonale Parapro teinämie bei Virusinfektion und maligner lymphatischer Erkrankung. Dtsch. Med. Wschr. 110, 837-840 (1985)

26. HEWELL, G. M., ALEXANIAN, R.: Multiple myeloma in young persons. Ann. of Intern. Med. 84, 441-443 (1976).

27. HITZIG, W. H.: Paraproteinämien im Kindesalter. Protides of the Biol. Fluids. Proc. of the XVIII. Collogium Bruges (1970)

28. HOBBS, J. R.: Bence-Jones Proteins Essays. Med. Biochem. 1, 105 (1975). 29. HOSLEY, H. F.: M-proteins, plasmocytosis and cancer. Cancer 20, 295 (1967). 30. JOHANNSON, S. G., HÖGMANN, C. F., KILLANDER, J.: Quantitative immun globulin determination. Acta Path. Microbiol. Scand. 74, 519 (1968).

31. KLEIN, G., KLEIN, E.: Evaluation of tumors and the impact of molecular oncology. Nature 315, 190 (1985)

32. KLEMM, D., GRUSNICK, D., WEINREICH, J. et al.: Paraproteinämie und plasma retikuläre Zellproliferation bei Polycythämia vera. Acta Haemat. 38. 240 (1967).

33. KRAPF, F.: Zirkulierende Immunkomplexe. In: Thomas, L. (ed.) Labor und Diagnose. Die Medizinische Verlagsges. Marburg, 571 (1984).

34. KRUSE, K., STEIN, H., HITZIG, W. H. et al : Lymph node plasmacytoma in a child: clinical and immunological findings with special reference to $\mathrm{S}-\mathrm{Ig}$ and complement receptor subtypes. Eur. J. Pediatr. 129, 239-257 (1978).

35. LINDQUIST, K. J., RAGAB, A. H., OSTERLAND, C. K.: Paraproteinemia in a child with leukemia. Blood XXXV, 213 (1970).

36. MAEDA, K., ABESAMIS, C., KUHN, L. M. et al.: Multiple myeloma in childhood: Report of a case with breast tumors as a presenting manifestation. Amer. J. Clin. Pathol. 60, 552-558 (1973).

37. MAUCH, H., HAMMER, H. J.: Die differenzierende Diagnostik monoklonaler und polyklonaler Immunglobuline mit der Immunfixation. Lab.med. 5, 227-230 (1981). 38. PETER, H. H., BARTHOLOMÄUS, C., MEYER ZU SCHWABEDISSEN, H. et al: Klinische und immunologische Befunde bei Patienten mit variablem Immundefektsyndrom (CVID). Verh. Disch. Ges. Inn. Med. 86, 1458 (1980).

39. PICK, A. J., VERSANO, J., SCHREIBMANN, S. et al.: Agammaglobulinemia, plasma cell dyscrasia and amyloidosis in a 12-year-old child. Am. J. Dis. Child 131, 682 (1977).

40. PORTER, F.: Multiple myeloma in a child. J. Pediatr. 62, 602-604 (1963)

41. PORUSH, J. G., GRISHMAN, E., ALTER, A. A. et al.: Paraproteinemia and cryoglobulinemia associated with atypical glomerulonephritis and the nephrotic syndrome. Amer. J. Med. 47, 957-964 (1969).

42. PUDILL, R.: Immunglobulinuntersuchungen im Liquor von Patienten mit multipler Sklerose (MS). Probleme der Diagnostik. Ärztl. Lab. 31, 297-302 (1985).

43. PUDILL, R.: Multiple Sklerose: Ausschlußdiagnostik durch Liquorprotein-Profil. Diagnose u: Labor 36, 28-34 (1986).

44. PUDILL, R., TARKKANEN, P.: Bi- und triklonale Gammopathien - Klinik und Diagnostik. Lab.med. 10, 249-257 (1986).

45. PUDILL, R., TARKKANEN, P.: Monoklonale und polyklonale Gammopathien bei Personen mit Human Immundeficiency Virus (HIV)-Infektion. Lab.med. 11, 101-107 (1987)

46. PUDILL R BETTGES G METZ K.Verlaufsbeobachtung bei einem extramedul lären Plasmozytom mit gesteigerter Anaplasie und Verlust immunelektrophoretisch nachweisbarer Lambdakettenbildung. Immunität und Infektion 10, 115-117 (1982). 47. RADL, J., DOOREN, L. J., MORELL, A., SKVARIL, F. et al.: Immunoglobulins and transient paraproteins in sera of patients with Wiskott-Aldrich syndrome. Clin. Exp. Immunol. 25, 256-263 (1976).

48. SCHNEIDERBAUR A. Ein Fall von Lebercirnhose mit einer beta-Globulin-Plasmocytose. Wien. Med. Wschr. 112, 36 (1962).

49. SCHULTE-WISSERMANN, H., GARDILCIC, S.: Die Rolle des Thymus in der Entwicklung der Immunkompetenz. Monatsschr. Kinderheilkd. 129, 378-390 (1981) 50. SCHULTE-WISSERMANN, H.: Immunologische, allergische und verwandte Krankheiten. In: Schulte, F. J., Spranger, J. (eds.) Lehrbuch der Kinderheilkunde, 25. Aufl. Gustav Fischer Verlag. Stuttgart, 461 (1985).

51. SELIGMANN, M., FUDENBERG, H., GOOD, R.: A proposed classification of primary immunologic deficiencies. Amer. J. Med. 45, 817 (1968).

52. SHIRLEY, L. R., BUCKLEY, R. H., BOROWITZ, M. J. et al.: Monoclonal immunoglobulin-secreting lymphoma in a patient with severe combined immunodeficiency disease. Clin. Exp. Immunol. 48, 666-674 (1982).

53. SPENLER G. A ROULET, D. L A RICCl, C.: Paraproteinämien bei chronischer Lymphadenose. Schweiz. Med. Wschr. 91. 984 (1961).

54. THOMAS, L.: Hypo- und Agammaglobulinämien. In: Proteindiagnostik, Diagnose und Therapiekontrolle. Behringwerke AG (ed.), 42 (1982).

55. TICHYY, M., HRNCIŔ, Z., URBANKKOVA, J.: Transient IgM-Lambda paraprotein in a 15-month-old child. Clin. Chim. Acta 70, 201-204 (1976).

56. TSUNG, S. H.: Monoclonal gammopathy associated with multiple sclerosis. Am Clin. Lab. Sci. 8, 472-475 (1978).

57. WEINAND, H. A., HABERLAND, K., PUDILL, R. et al.: Niereninsuffizienz be Leichtketten-Paraproteinämie. Med. Welt 29, 346-349 (1978).

58. ZAWADZKI, Z., AIZAWA, Y., KRAJ, M. A. et al.: Familial Immunopathies. Cancer 40. 2094-2101 (1977).

59. ZIEGLER, J., DREW, W. L., MINER, R. C. et al.: Outbreak of Burkitt's-like lymphoma in homosexual men. Lancet 2, 631-633 (1982).

60. ZIEGLER, J., BECKSTEAD. J. A., VOLBERDING. P. A. et al.: Non-Hodgkin's lymphoma in 90 homosexual men. N. Engl. J. Med. 311. 565-570 (1984).

Anschrift für die Verfasser:

Dr. Rainer Pudill

Labor Dr. med. P. Tarkkanen

Bismarckplatz 4

4050 Mönchengladbach 1 